\section{La Casa dell'abate a San Giovanni in Persiceto: Indagini conoscitive e questioni operative per la conservazione di un "edificio-fossile»}

\section{The Abbot's House in St. Giovanni in Persiceto: Cognitive surveys and operative matters for the preservation of $a$ «Fossil-building»}

Angela Paola SQUassina

Università IUAV di Venezia*

\begin{abstract}
Riassunto
La Casa dell'Abate a San Giovanni in Persiceto (Bologna) è un rarissimo manufatto medioevale a struttura mista in legno e muratura, uno dei pochi superstiti in Italia, probabile sede dell'Abate del Monastero di Nonantola. Si tratta di un «edificio-fossile», oggetto di grande interesse data la rarità, l'antichità della struttura e delle modalità costruttive, nonché per la presenza di una stratificazione quasi millenaria.

La Casa dell'Abate è stata infatti sottoposta ad un lento ma continuo processo di trasformazione per sostituzione graduale degli elementi lignei con muratura, che lo ha condotto da una condizione di edificio-fondaco a carattere non residenziale, verso un assetto prevalentemente abitativo.

A ciò si aggiunge una generale condizione di ridotta leggibilità stratigrafica, dovuta ad un intervento risalente agli anni Sessanta, che ha comportato profonde trasformazioni strutturali ed una generale alterazione delle superfici esterne sistematicamente rinnovate, con conseguente modifica della percezione di antichità dell'insieme.

Una condizione che suggerisce alcune riflessioni sul rapporto fra leggibilità stratigrafica e conservazione dell'autenticità. In un edificio fortemente alterato, viene drasticamente ridotta la capacità risolutiva dello strumento stratigrafico, di cui diventa necessario ricalibrare codici e modalità applicative. Tuttavia, da questa necessità di continuo adattamento a diverse condizioni di lettura, risulta un'articolazione della stratigrafia - qualora inclusa organicamente nella concezione del progetto di restauro - e la leggibilità stratigrafica può divenire anche prezioso indicatore delle alterazioni indotte e criterio tecnico-culturale di orientamento del progetto, come tensione ideale verso il controllo delle trasformazioni nell'intervento. Che si configura qui in termini minimali e rispettosi delle testimonianze superstiti frammentate e della loro leggibilità stratigrafica.
\end{abstract}

Parole chiave: stratigrafia, leggibilità stratigrafica, edifici medioevali a struttura lignea, conservazione, autenticità.

\section{Abstract}

The Abbot's House in St. Giovanni in Persiceto (Bologna) is a «fossil-building», one of the few surviving timber-framed buildings in Italy, probably the seat of the Abbot of the monastery of Nonantola.

It is very interesting because of its rarity, age, building type and the presence of an almost millenarian stratigraphy.

The Abbot's House has been subjected to a slow but continuous

transformation process - through a gradual replacement of wooden elements by masonry - modifying its storehouse function for a prevalently residential one.

Restoration works in the 1960s deeply modified the structural frame and renewed the external surfaces, transforming thus the old image of the building and making difficult its archaeological analysis.

This circumstance makes to think about the relationship between stratigraphic legibility and preservation of material authenticity. Reliability of stratigraphic instrument becomes reduced in such a modified building, being required a different use of stratigraphy by adapting its codes and modes to the study case.

The adaptation to different recording conditions gives stratigraphy a different and more articulated role - if it is organically included in the preservation project - and stratigraphic legibility may become a precious alteration index and a technical and cultural project criterion. It becomes a control of transformations, leading to the idea of a minimal intervention and respect toward the fragmentary survivals of past times and of their stratigraphic legibility.

Key words: stratigraphy, stratigraphic legibility, medieval timber-framed buildings, conservation, authenticity.

\section{INTRODUZIONE: LEGGIBILITÀ STRATIGRAFICA E CONOSCENZA MATERIALE}

La lettura stratigrafica è possibile in condizioni ottimali su superfici interessate da un blando degrado erosivo e non eccessivamente turbate da interventi di radicale modificazione, ovvero da depositi o ricoperture che possano impedire la visibilità delle caratteristiche tessiturali e dei contatti.

Le condizioni reali si distanziano in modo più o meno marcato da questo stato di leggibilità ottimale. Se a fronte di una leggibilità parziale non può che esserci interpretazione parziale o dubitativa, per ogni edificio varia e va quindi ritrovata la capacità di risoluzione dello strumento stratigrafico, di cui diventa necessario ricalibrare ogni volta l'uso, riadattandone i codici e le modalità applicative.

Nel caso della Casa dell'Abate a San Giovanni in Persiceto (Bologna), un edificio a struttura lignea di origine medioevale, la leggibilità stratigrafica risulta turbata da una condizione di degrado diffuso, dovuta ad uno stato di abbandono prolungato ma anche dagli esiti di un restauro che ha comportato una serie di demolizioniricostruzioni, i cui effetti assumono la connotazione di peculiari forme di degrado, misurabili soprattutto in termini di alterazione del carattere dell'edificio e di perdita di materia antica.

A fronte della parzialità conoscitiva dovuta alla penuria di notizie storiche disponibili, nonostante la rarità ed il forte significato simbolico dell'edificio ${ }^{1}$, l'ente proprietario (il Consorzio dei Partecipanti di San Giovanni in Persiceto) ha attivato una serie di rilievi ed analisi basati sulla lettura della materia del manufatto e dei segni stratificati ${ }^{2}$.

Svincolata da finalità operative immediate, seppure in vista di un'iniziativa di tutela e valorizzazione, l'indagine è stata condotta prevalentemente con tecniche archeometriche e si è sviluppata come ricerca indiziaria delle parti

1 Lorigine della Casa dell'Abate è stata oggetto di indagine in passato ma non risulta essere stata ancora chiarita. L'ipotesi più accreditata vi individua la sede rappresentativa dell'Abate di Nonantola, luogo di riscossione di tributi. Un'ipotesi avvalorata dalla localizzazione esterna all'antico nucleo abitato, in corrispondenza di un polo della via che nell'alto Medioevo collegava direttamente S. Giovanni con il monastero, che ne mantenne la proprietà fino al XVIII secolo. L'edificio è la testimonianza materiale degli stretti rapporti intercorsi fra l'Abbazia di Nonantola e la comunità persicetana, riunita nella Partecipanza, l'istituto che cura tuttora la gestione dei terreni di S.Giovanni, donati dai Duchi di Persiceto all'Abbazia.

${ }^{2}$ Il lavoro è stato svolto da chi scrive con la direzione scientifica del Prof. F. Doglioni dell'Università IUAV di Venezia e con la collaborazione degli architetti S. Mantovani e D. Zaghi, cui si devono le elaborazioni digitali e la modellazione tridimensionale. Sulla base di questa indagine ed a specificazione di ulteriori aspetti, si è sviluppata un'iniziativa di collaborazione istituita fra la Partecipanza e lo IUAV, facoltà di Architettura, che ha dato luogo ad una tesi di laurea ed una serie di esercitazioni didattiche nell'ambito del Laboratorio Integrato di Restauro, Corso di Laurea Specialistica in Conservazione dell'Architettura (Prof. F. Doglioni, Prof. F. Focacci, Prof. F. Laner). 

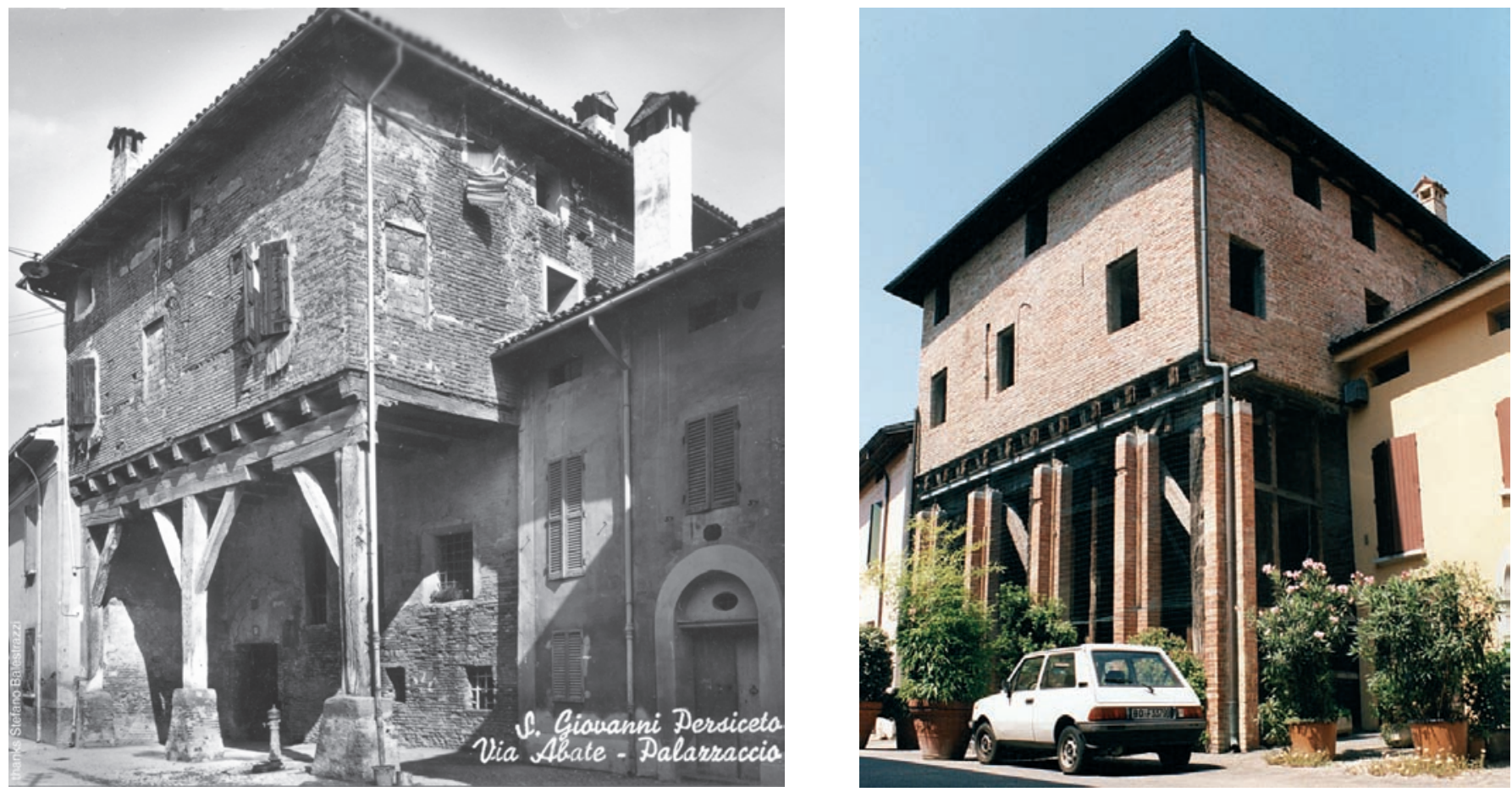

Fig. 1 A-B. La Casa dell'Abate a S.Giovanni in Persiceto in una immagine precedente l'intervento (per gentile concessione del Sig. Stefano Balestrazzi e del Consorzio dei Partecipanti di S.Giovanni in Persiceto) e nella configurazione attuale

superstiti dell'assetto iniziale, orientata a far luce sui suoi caratteri costruttivi, sulle modifiche architettoniche e dei modi d'uso. Un vero e proprio cantiere di conoscenza, basato sulla possibilità di decifrare le tracce nel «documento di pietra", costituito dalla materia dell'edificio con le sue trasformazioni. Pur senza sottovalutare l'importanza di un processo integrato fra fonti dirette ed indirette, la carenza di dati documentali ha fatto emergere in questo caso il ruolo conoscitivo del rapporto diretto con la materialita ${ }^{3}$ — intesa come «dato esistenziale» ${ }^{4}$ dell' architettura, luogo di sedimentazione dei segni del tempo - e l'importanza degli aspetti di registrazione e trasmissibilità delle informazioni.

Il peculiare carattere dell'oggetto - un «edificiofossile», che non ha avuto processi di rinnovo organico e sistematico per molto tempo, ma adattamenti parziali e spesso poveri, seguiti da alcune forti trasformazioni recenti, che ne hanno ridotto la consistenza, modificandone fortemente anche la percezione - suggerisce alcune riflessioni sul rapporto fra degrado, leggibilità stratigrafica e percezione dell'autenticità materiale.

\footnotetext{
${ }^{3}$ Questo aspetto è stato più volte sottolineato da Roberto Parenti, cui si devono alcuni dei primi significativi esempi di lettura stratigrafica in ambiti operativi di restauro, ed alimentato da esperienze didattiche e professionali maturate da Francesco Doglioni.

${ }^{4}$ Bellini, «La superficie», 1990.
}

Con le parole di Hugo:

«(...) Io chiedo, scordando le ore All'antica eco di quelle dimore

Ciò che resta della loro voce $(\ldots){ }^{5}$

In questo senso, il forte senso di antichità ancora trasmesso - pur nel degrado e forse in parte anche grazie al degrado - dalla struttura lignea del portico quasi fossilizzata e dalle superfici non rinnovate, induce alla necessità di graduare l'azione di contrasto al decadimento fisico ed orienta verso l'idea di un restauro minimale e di un utilizzo compatibile con la permanenza delle testimonianze superstiti frammentate e della loro leggibilità stratigrafica (figg.1a-b).

\section{LA «CASA DELL'ABATE»: FONTI PUBBLICISTICHE, CARATTERI COSTRUTTIVI E LETTURA DELLE TRASFORMAZIONI}

Allo stato attuale delle ricerche non è possibile stabilire una datazione assoluta su base documentaria, è tuttavia plausibile proporre una collocazione cronologica fra il XIII ed il XIV secolo, anche sulla base di una serie di analogie costruttive con edifici bolognesi più noti (Casa Isolani, Casa Grassi, Casa Serracchioli) (figg. 2 A-B). La tipologia edilizia è quella dell'abitazione medioevale a struttura

\footnotetext{
V. Hugo, «La bande noire», 1823.
} 

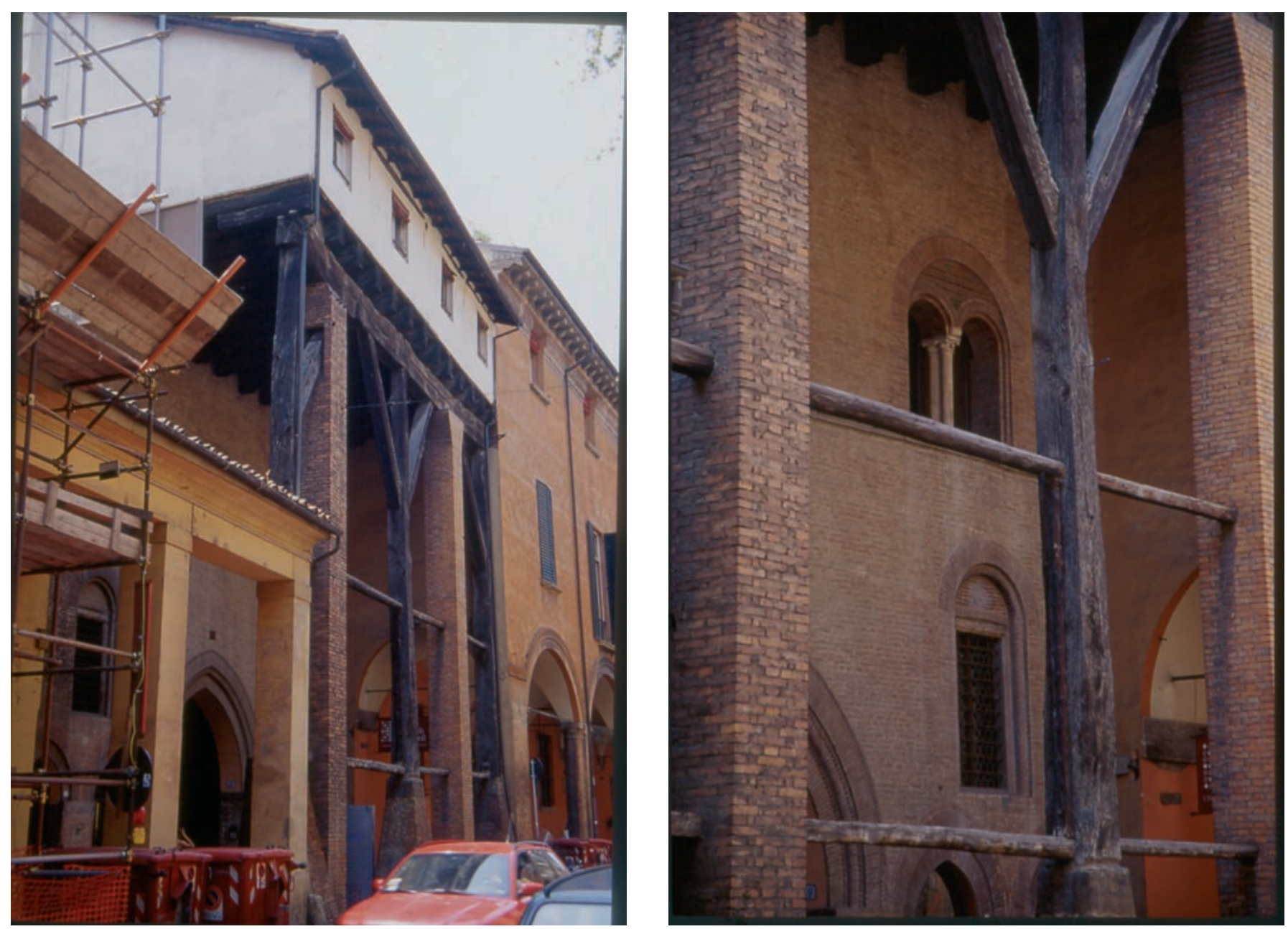

Fig. 2 A-B. Casa Isolani a Bologna, che rappresenta un riferimento tipologico, per l'analogia strutturale e di assetto complessivo

lignea, con portico a doppia altezza, sostenuto da stilate lignee (pilastri snelli con diagonali «a stampella») poggianti su plinti in muratura ${ }^{6}$ (figg. $3 \mathrm{~A}-\mathrm{B}$ ).

Si tratta di un manufatto caratterizzato da una stratificazione complessa e da peculiari caratteri costruttivi dovuti alla struttura mista, che implica di per sé un sistema di contatti legno-muratura di non facile decifrazione in termini stratigrafici, cui si aggiunge una generale condizione di ridotta leggibilità, dovuta ad un intervento a forte componente strutturale (figg. 3 C-D), risalente agli anni Sessanta, che ha comportato profonde trasformazioni anche alle superfici.

${ }^{6}$ Rappresentativo del contesto urbano medioevale ed ormai rarissimo, questo genere è il risultato di un perfezionamento tecnico-costruttivo di precedenti abitazioni a sporto e di una più stretta regolamentazione dell'uso dei suoli pubblici, che ebbe luogo nel corso del Duecento in Italia. L'edificio di San Giovanni diventa così uno dei pochi esemplari superstiti in area emiliana, emblema dello stretto rapporto esistente nel Medioevo fra insediamento urbano, assetto sociale-produttivo e caratteri costruttivi delle abitazioni (si veda F. Bocchi, A. Benati, 1990 e G. Rivani, 1966).
L'aspetto che immediatamente colpisce l'osservatore, alla luce delle nozioni sull'antica origine della Casa dell'Abate, è oggi costituito da una sorta di «sdoppiamento», se così si può definire il contrasto fra l'aspetto esterno omogeneo, quasi moderno, conferito dal restauro ed il degrado delle parti antiche. Un insieme dal carattere contraddittorio, misurabile quasi esclusivamente in termini percettivi, cioè di diverso grado di significatività-»emissività» delle superfici, in relazione alla diversa profondità temporale che le connota. Una condizione che suggerisce quesiti di natura stratigrafica ed induce ad un diverso uso dello strumento stratigrafico vero e proprio, oltre che ad una diversa lettura del degrado.

\section{ADATTAMENTO DEI CODICI DI LETTURA AL DIVERSO GRADO DI LEGGIBILITA' STRATIGRAFICA}

Una conseguenza immediata è una diversa capacità risolutiva dello strumento stratigrafico, in quanto operazioni di sostituzione comportano la separazione fisica fra le parti 

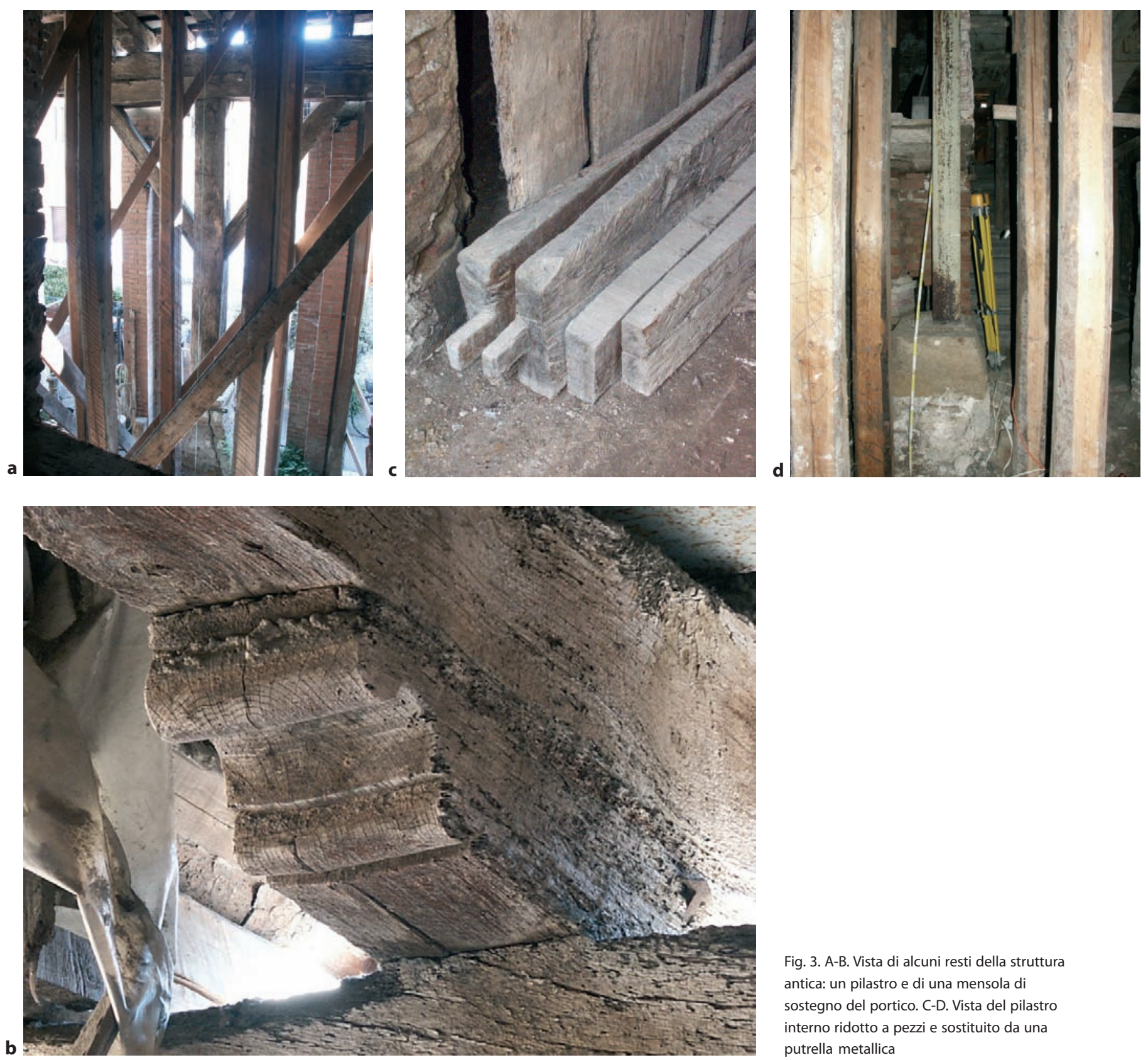

Fig. 3. A-B. Vista di alcuni resti della struttura antica: un pilastro e di una mensola di sostegno del portico. C-D. Vista del pilastro interno ridotto a pezzi e sostituito da una putrella metallica

superstiti, con conseguente riduzione drastica del numero di nessi stratigrafici significativi.

La formazione di sezioni fotografiche complessive, ottenute inserendo nei grafici di rilievo i fotomosaici delle superfici relative, ha posto in evidenza i diversi frammenti osservati ai vari piani, consentendone la correlazione alle strutture architettoniche più antiche, inglobate nelle murature successive (figg. 4 e $11 \mathrm{~A}-\mathrm{B}$ ).

Lo strumento stratigrafico è stato inoltre integrato da un censimento delle murature, localizzate in pianta e documentate in un abaco, che ha permesso di correlare ogni tipo murario ad una singola fase costruttivo-trasfor- mativa, ovviando in parte alla condizione di ridotta leggibilità stratigrafica (fig. 5).

\section{a) Necessità' di una lettura pseudo-stratigrafica o per comparazione indiretta nei contesti trasformati (figg. $6 \mathrm{~A}-\mathrm{B}$ )}

Le facciate sono state sottoposte ad interventi di parziale ricostruzione e ad un completo rifacimento dei giunti, che hanno reso incerta la decifrazione delle tracce, in particolare dei bordi stratigrafici. Si è reso necessario costruire uno strumento di lettura misto, ricorrendo ad un'osservazione "pseudo-stratigrafica», in parte diretta (sulla facciata nella 

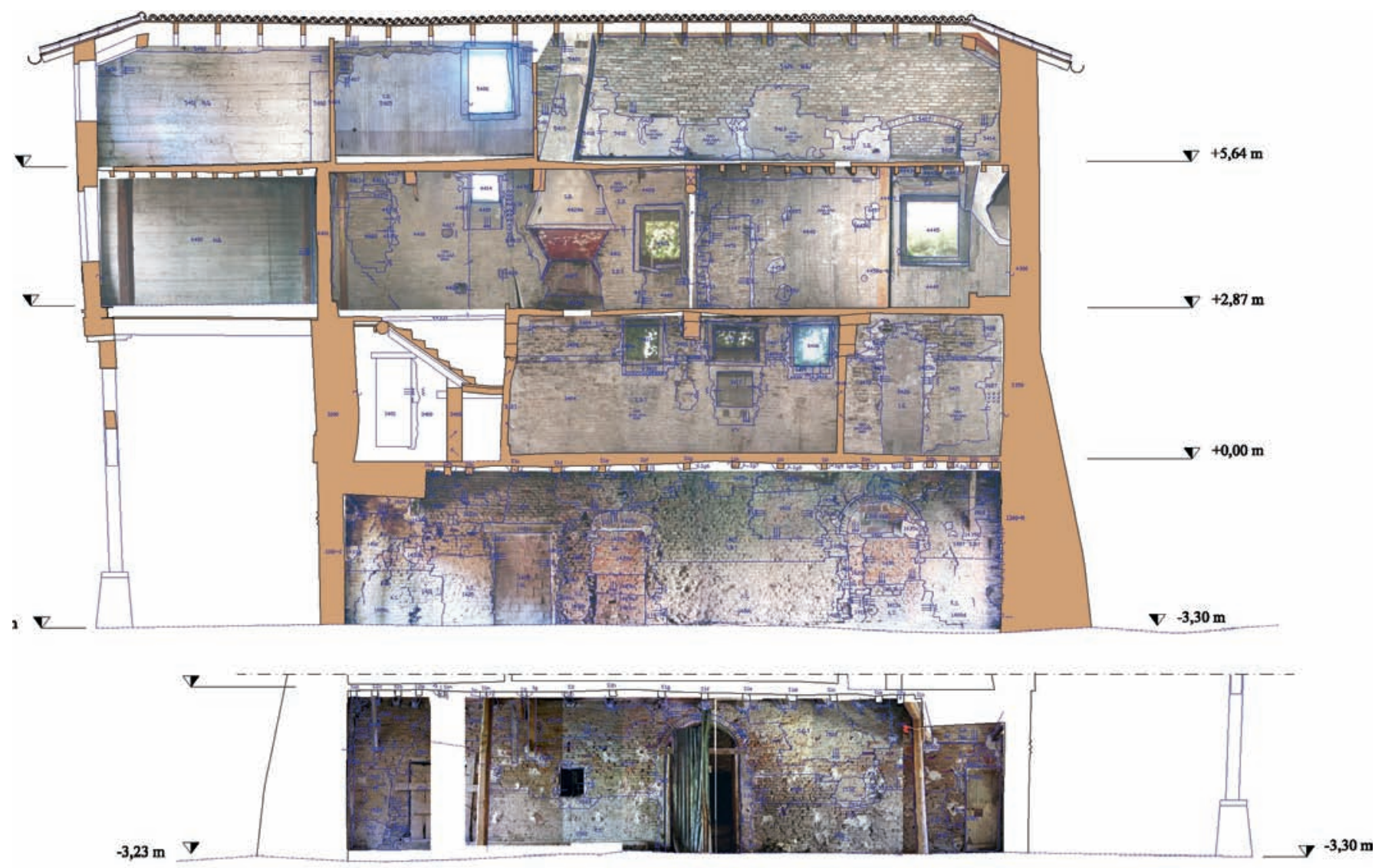

Fig. 4. Le sezioni fotografiche sono un modello descrittivo in grado di compendiare diversi tipi di informazioni e di rappresentare al contempo il dato dimensionale e la qualità materica delle superfici

configurazione attuale), in parte indiretta, utilizzando come supporto una fotografia precedente l'intervento di rinnovo, per un successivo confronto fra i dati ricavati dalle due letture.

Si è trattato di uno studio comparato che ha richiesto l'adeguamento degli strumenti esistenti e la costruzione di codici di lettura ad hoc, comprensivi di simboli utili ad indicare il genere di trasformazioni individuate (parti conservate senza trasformazioni, parti conservate con alterazioni, parti riconfigurate/ricostruite, parti aggiunte e parti rimosse; parti nascoste e parti/elementi riemersi; parti non riconoscibili).

Le superfici rinnovate all'esterno ma leggibili all'interno hanno permesso di trasferire le informazioni fornite da evidenze stratigrafiche sul corrispondente paramento non leggibile. Solo grazie all'intreccio delle informazioni contenute nelle due fonti distinte - documento storico, documento materiale - è stato possibile ipotizzare un assetto iniziale delle facciate con monofora centrale sui fronti principali ed aperture in angolo su quelli laterali.

\section{b) Contributo dei nessi stratigrafici alla conservazione dell'autenticità}

La completa sostituzione dei giunti riduce drasticamente le potenzialità della stratigrafia limitando l'osservazione possibile alle discontinuità tessiturali e di configurazione. Soltanto l'osservazione del corrispondente paramento interno non restaurato, in particolare delle malte nei giunti, permette di dare significato stratigrafico a discontinuità percepibili solo per forma sul paramento rinnovato (figg. 7 A-B).

Frammenti di due aperture ad arco correlabili alla muratura iniziale grazie all'identicità della malta ed incompatibili con gli attuali livelli di solaio, fanno constatare come permanenza di materia non alterata e leggibilità stratigrafica siano strettamente correlate. Una porzione anche ridotta di arco, che abbia mantenuto la leggibilità dei rapporti fra muratura, apertura, orizzontamento, consente infatti la possibilità di lettura di stratificazioni anche complesse, cioè di stabilire relazioni cronologiche attendibili fra le diverse parti che compongono un intero contesto tridimensionale. 

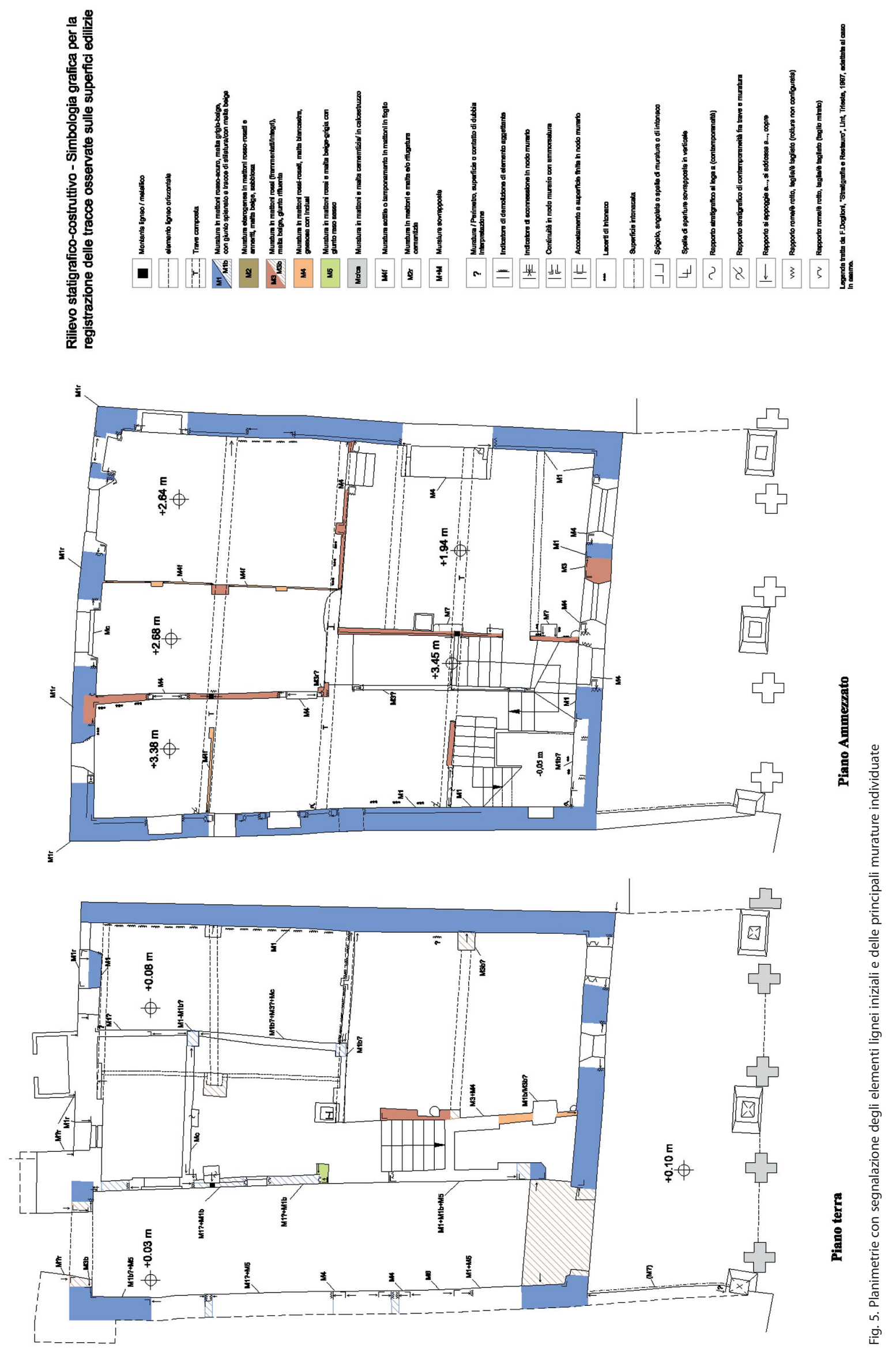

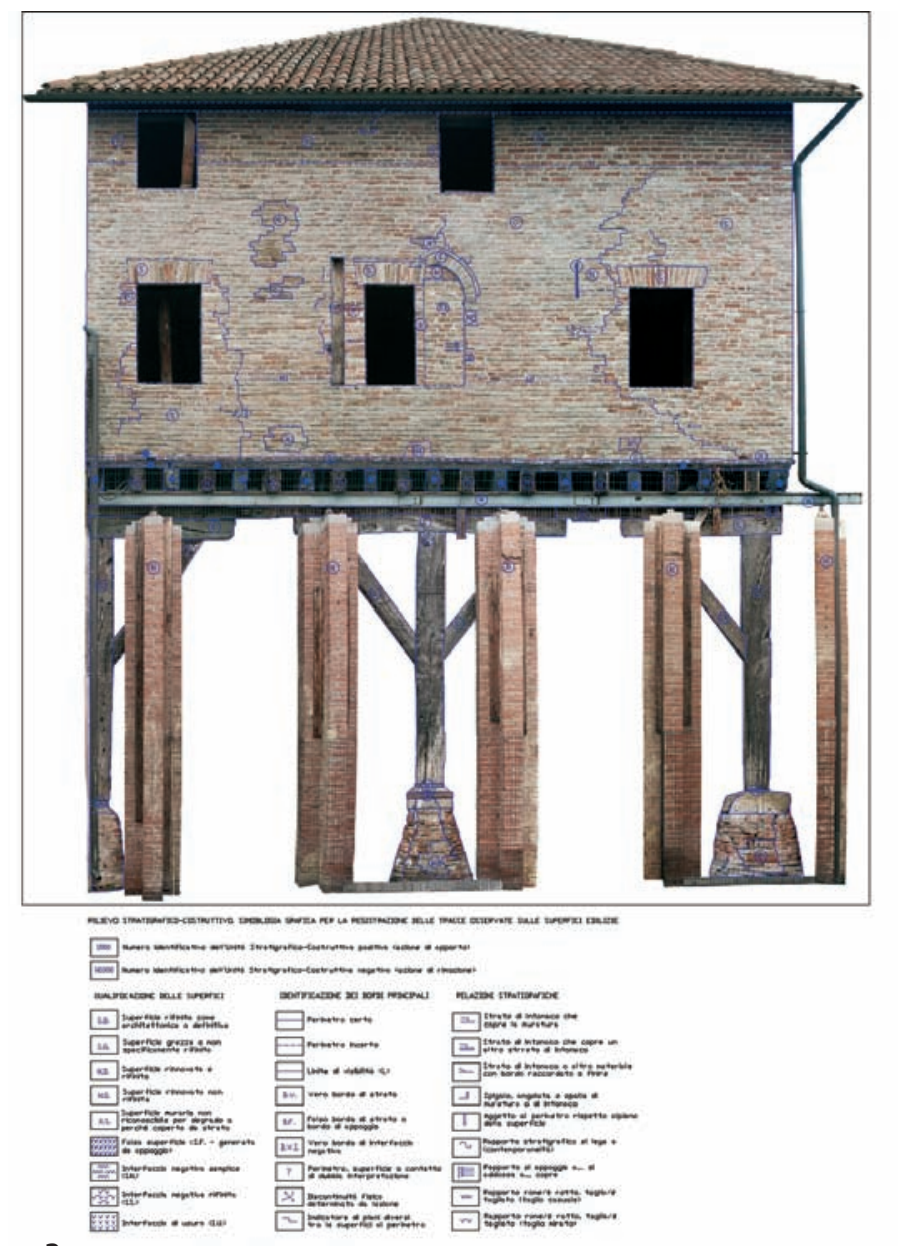

a

E' chiaro cioè il ruolo stratigrafico del frammento, ancorché dotato dei propri nessi, nell'attestare la permanenza dell' "autenticità per relazione» ${ }^{7}$ nel manufatto, che si perde invece nel caso di una compiutezza formale che sia privata delle relazioni con il proprio contesto materialestratigrafico.

Nel caso in esame il metodo stratigrafico ha subito un continuo adattamento allo stato dei singoli ambiti, dimostrando una certa duttilità di impiego, che va però calibrata al diverso grado di incertezza di volta in volta ritenuto accettabile. A fronte di un aumento del margine di dubbio diventa necessario mantenere aperte alcune questioni interpretative.

Rimane infatti parzialmente irrisolta la questione della validità del trasferimento di informazioni fra due superfici diversamente connotate, se non che la parete rinnovata funge da suggerimento, sulla base di forme compiutamente percepibili, alla controparete interna non restaurata; ma

\footnotetext{
Il concetto di «autenticità per relazione», indispensabile a comprendere l'importanza della leggibilità dei nessi stratigrafici per la conservazione dell'autenticità di un manufatto, emerge in F. Doglioni, «Importanza dell'intonaco», 1993.
}

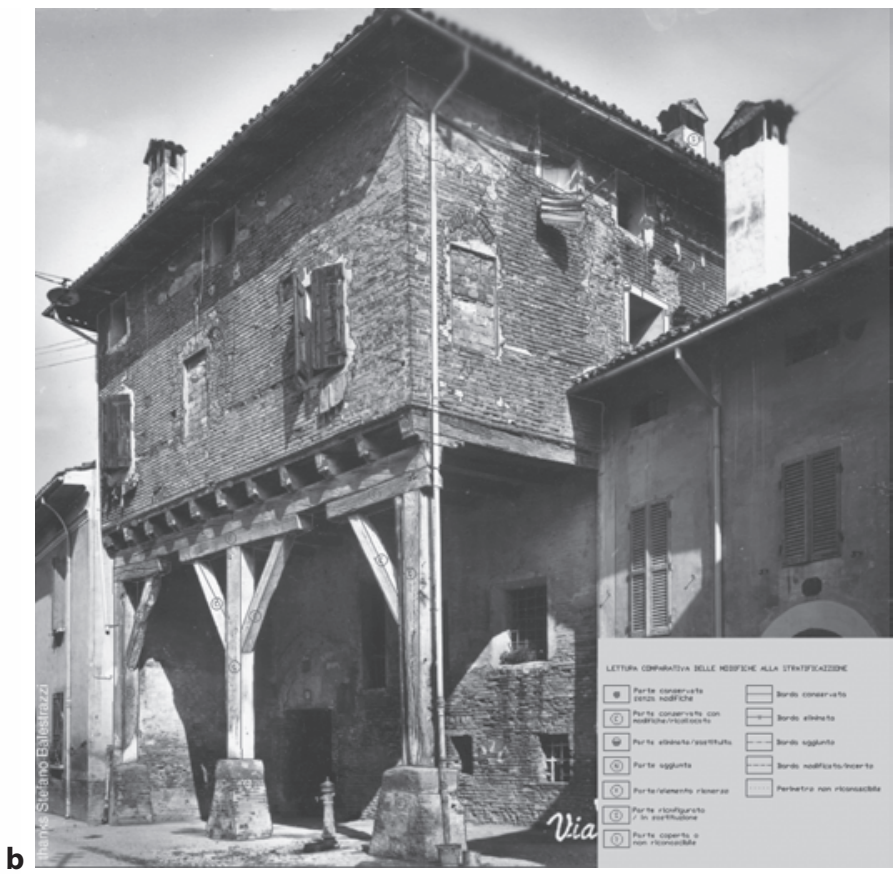

Fig. 6 A-B.- Dalla comparazione fra risultati delle osservazioni sulla fotografia storica ed esisti della lettura diretta delle superfici sono emersi gli indizi di un innalzamento dell'edificio e di un processo di smontaggio e rimontaggio degli elementi lignei di facciata

solo quest'ultima si presta ad una lettura stratigrafica vera e propria. I due paramenti sono in qualche modo evocativi l'uno rispetto all'altro ma nulla è possibile stabilire riguardo alla solidarietà tridimensionale, perduta con il restauro.

D'altra parte, pur nell'evidenza di questi limiti metodologici ed interpretativi, si colgono ulteriori potenzialità dello strumento stratigrafico nel controllo delle trasformazioni, dove la riduzione o l'alterazione delle condizioni di leggibilità stratigrafica diviene misura dell'irreversibilità indotta dagli interventi di restauro. ${ }^{8}$

\section{c) La «microstratigrafia» dei giunti di malta}

La facciata interna al portico non ha subito alcun intervento e, pur nel degrado che offusca le caratteristiche cromatiche e tessiturali delle superfici, conserva le tracce materiali e formali più significative per la comprensione dell'edificio.

\footnotetext{
${ }^{8}$ In questo senso trova ulteriore conferma la tesi di fondo contenuta in $\mathrm{F}$. Doglioni, «Stratigrafia e Restauro», 1997, che suggerisce un ampliamento sostanziale del ruolo della stratigrafia da strumento analitico con finalità prettamente conoscitive a struttura concettuale, che assume significati molteplici e differenziati nel corso dell'iter progettuale, durante le fasi di cantiere ed anche dopo l'intervento.
} 

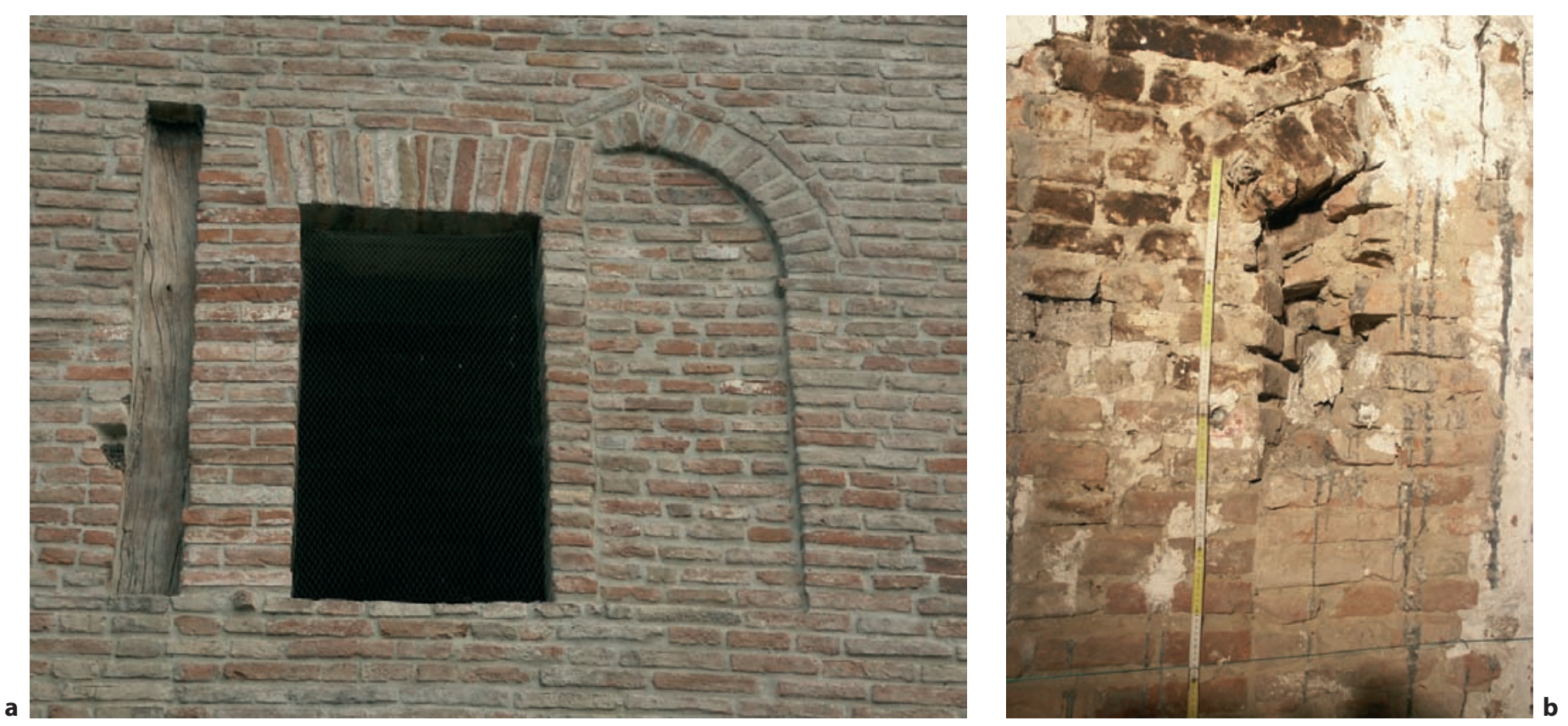

Fig. 7 A-B. Un diverso grado di leggibilità distingue le aperture restaurate da quelle non manipolate, le prime distinguibili solo per forma, le seconde leggibili stratigraficamente

L'attenzione stratigrafica cambia scala, scostandosi dal livello macroscopico, basato sull'osservazione delle unità stratigrafico-murarie ed elementi costruttivi, che solo un forte grado di permanenza consente, giungendo ad una sorta di «microstratigrafia», dove la capacità risolutiva è affidata al dettaglio, al giunto di malta, l'elemento più minuto e vulnerabile della stratificazione.

Nonostante il pessimo stato di conservazione delle malte, è stato proprio il giunto a fornire indicazioni sulla successione stratigrafica delle diverse parti, come l'arco d'ingresso ad ogiva e cornice in laterizio decorato o la monofora centrale, che era probabilmente un'apertura da carico (figg. 8 A-B). Inoltre, lacerti di finitura hanno confermato l'ipotesi di configurazione iniziale, con muratura in mattoni a vista e giunto stilato.

Ma il degrado da erosione, che per certi versi facilita la lettura stratigrafica, oltrepassati certi limiti la ostacola fortemente, quando aggredisce la malta, che costituisce il legame fisico e stratigrafico fra gli elementi del paramento murario. Per contro, il completo rifacimento dei giunti comporta una notevole perdita di significato stratigrafico della muratura, per la cancellazione dei bordi di discontinuità.

Si pone quindi il problema operativo e concettuale della conservazione-integrazione di queste malte, alcune erose in profondità o addirittura disgregate, ma indispensabili al mantenimento della leggibilità stratigrafica e dell'autenticità materiale del complesso stratificato.

Ognuna di queste particolari condizioni ha fatto con- statare l'inefficacia e dunque l'inapplicabilità di un rigoroso metodo stratigrafico nei contesti più trasformati, dove si è reso necessario comprendere entro quali limiti fosse possibile attribuire un significato alle unità stratigrafiche allorché, private della leggibilità dei nessi stratigrafici reciproci, si riducevano a semplici "porzioni» di materia configurata.

Solo con questi limiti posti all'interpretazione è stato possibile ricostruire l'assetto dell'edificio ligneo iniziale e stabilire una sequenza delle principali fasi di trasformazione, individuandone almeno in parte il processo evolutivo.

L'edificio ha subito trasformazioni già in epoca remota, prevalentemente per sostituzione graduale degli elementi lignei — più vulnerabili al fuoco - con parti in muratura e da manufatto a carattere non residenziale e dotato di un limitato numero di aperture (edificio-fondaco), è stato gradualmente sottoposto ad una serie di partizioni verticali ed orizzontali ed a modifiche nell'assetto distributivo e funzionale, legate ad un uso prevalentemente abitativo (fig.9).

\section{Periodo A (XIII-XIV sec.) Edificio-fondaco a prevalente struttura lignea (fasi 0-1) (figg.10 A-B e 11 A-B)}

Edificio a struttura prevalentemente lignea o mista, più basso di quello attuale e con portico ed androne passanti, aperture ad arco e murature perimetrali in mattoni a vista $\mathrm{e}$ giunto stilato. La struttura lignea portante interna era formata da tre travi-capriate su pilastri a stampella a 

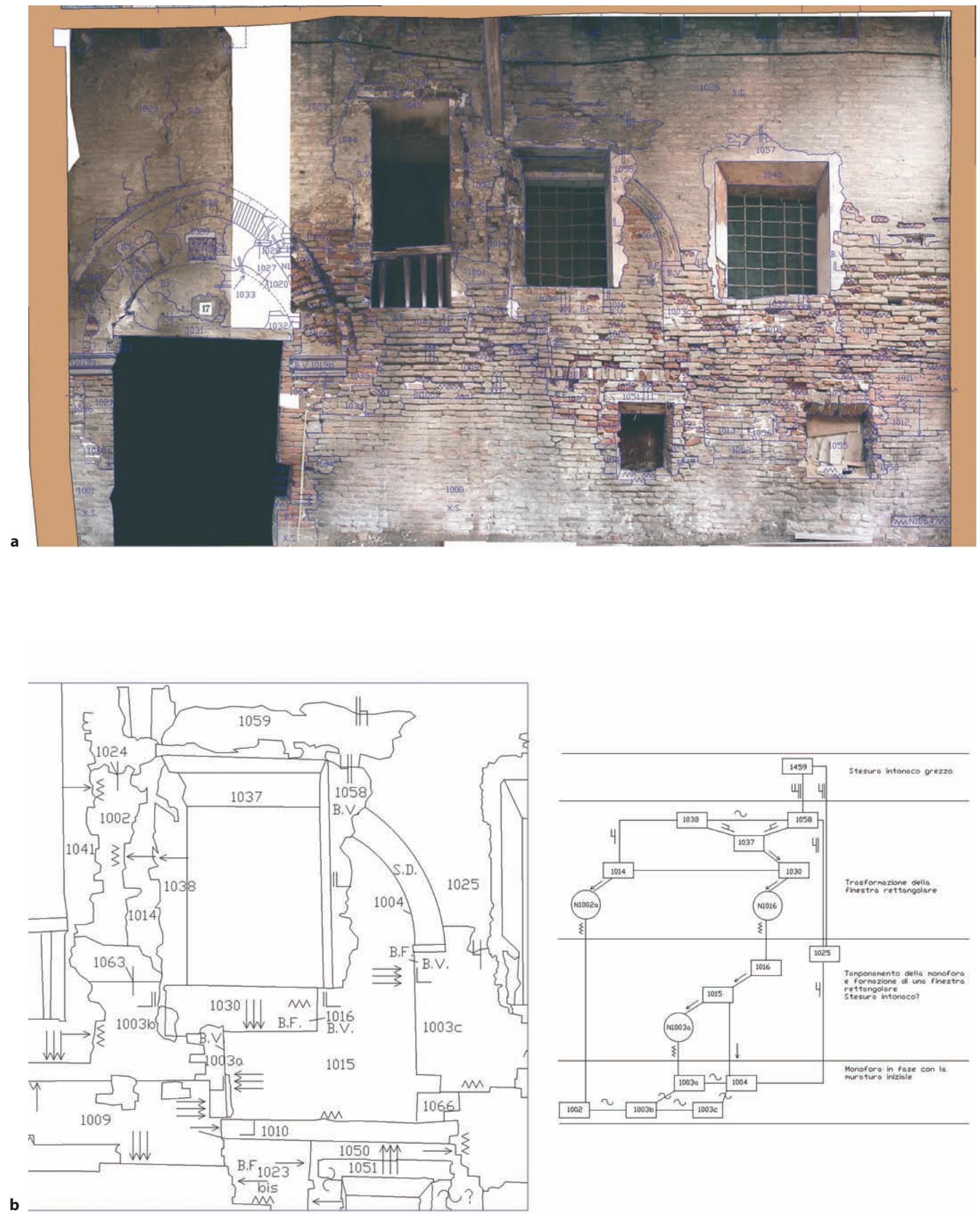

Fig. 8 A-B. Lettura stratigrafica e diagramma di Harris relativi alla monofora iniziale del portico 
PRINCIPALI FASI TRASFORMATIVE

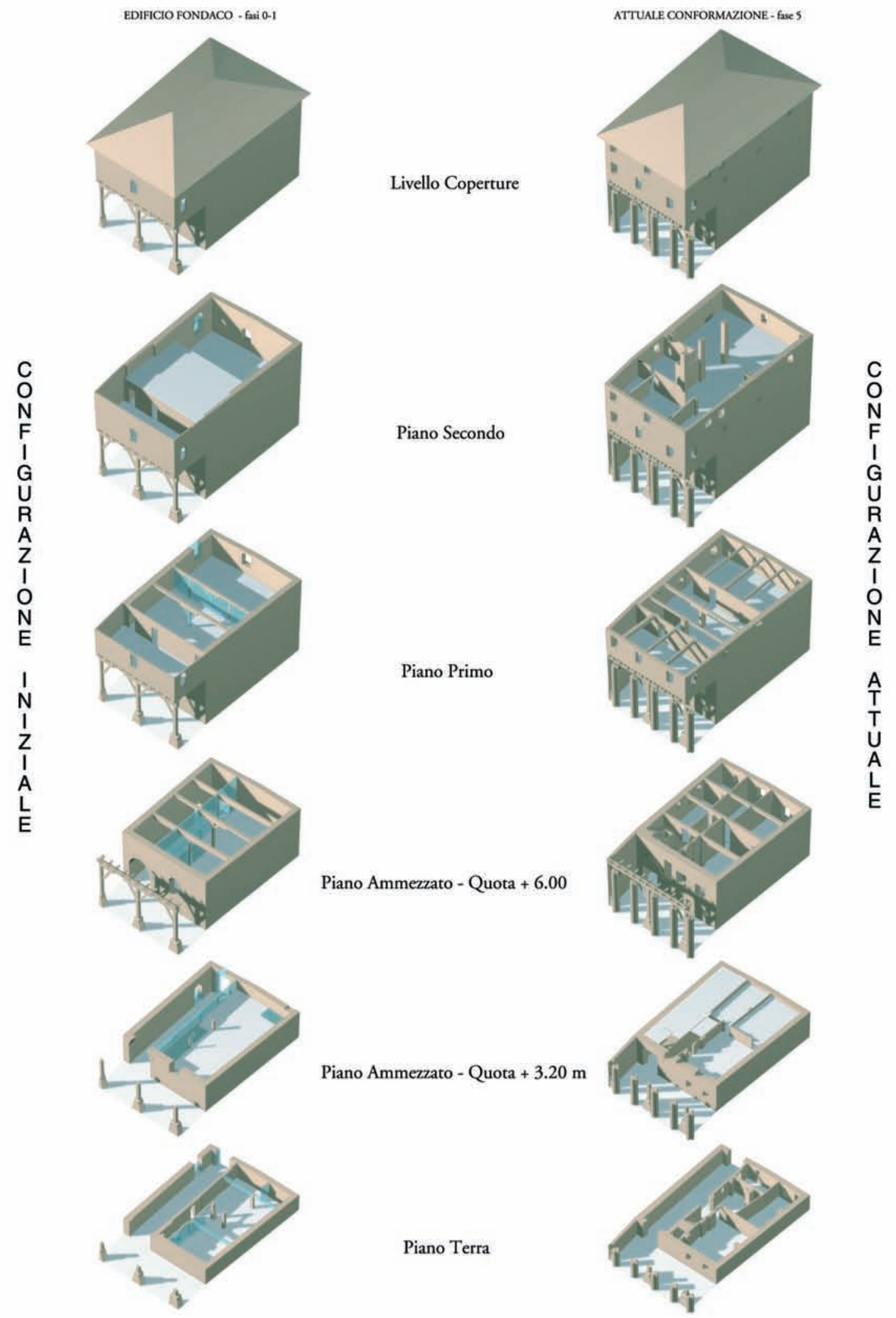

Fig. 9. Riepilogo schematico delle principali fasi trasformative individuate 

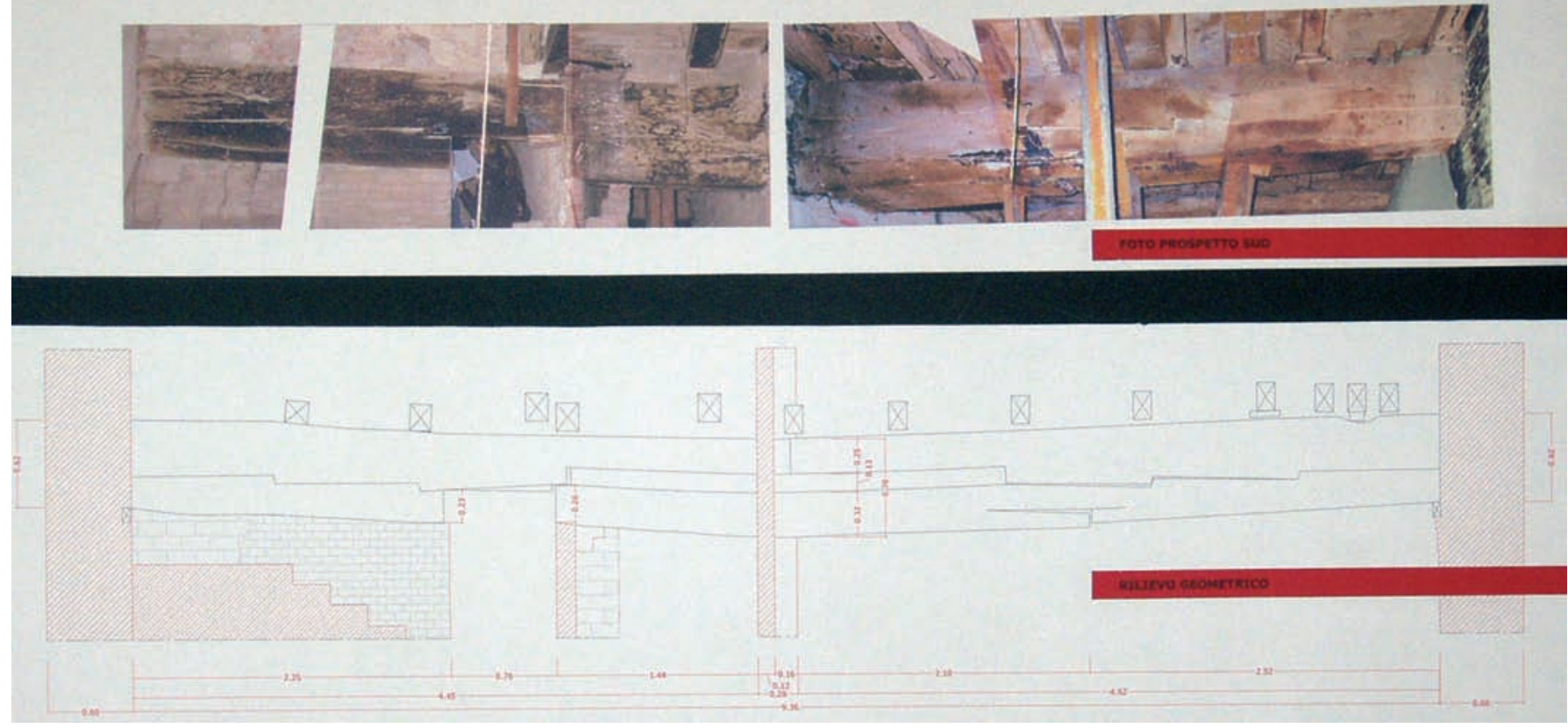

Fig. 10 A-B. Ricomposizione fotografica con ricostruzione grafica di una delle tre travicapriate interne (elaborato grafico dal Laboratorio Integrato di Restauro Architettonico, luav-Venezia, a.a.2002-2003, Prof. F. Doglioni, Prof. F. Focacci, Prof.F. Laner: Studenti: Marino, Onzani, Longo, Astolfi, Pellizzari, Dalla Mora, Battistello, Battaglia, Zane, Bagatella, Morisco, Beaugrand, Gerbelli, Marini, Ongaro, Marconi, Fracchetti, Fentè, Arana, Bala, Lèon, Garcia, Valina, Vicente) e modello dell'ipotetico assetto iniziale della struttura lignea interna (S.Mantovani, M.Zaghi)

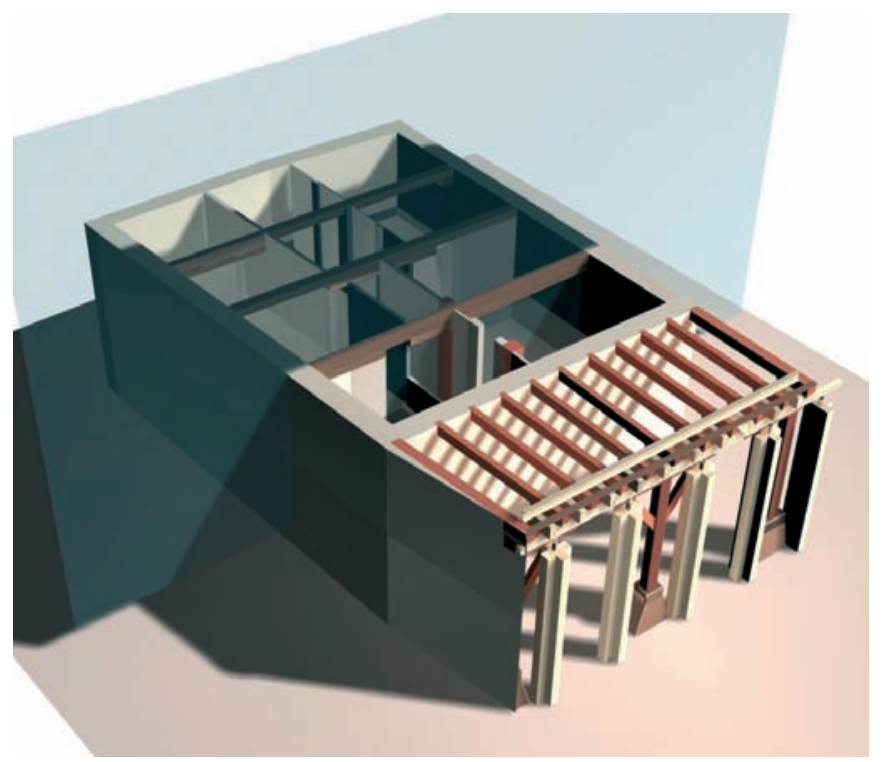

doppia altezza, con reme perimetrali e solaio ligneo con listelli. Alcuni montanti lignei con fori allineati, attualmente inglobati nelle murature, segnalano la presenza di un sistema di partizioni interne leggere.

\section{PerIOdo B (XIV-XVIII Sec.):}

\section{Edificio a struttura mista e destinazione residenziale} (fasi 2a,2b, 3) (figg.12 A-B)

Innalzamento dell'edificio con interventi di riassetto (formazione di una scala in muratura, documentata nel 1396 e di ambienti di dimensioni ridotte, dotati di aperture

rettangolari, che testimoniano un processo graduale ma continuo, già dalla fine del Trecento ${ }^{9}$, verso una struttura muraria ed una destinazione prevalentemente residenziale, con murature intonacate ed elementi di arredo fisso (camini, nicchie ed armadi a muro).

${ }^{9}$ La casa dell'Abate ricoprì un ruolo extra-territoriale almeno fino al suo inglobamento all'interno del centro cittadino, avvenuto intorno al 1306; a questo mutamento si potrebbe ascrivere anche una prima trasformazione costruttivo-funzionale. 

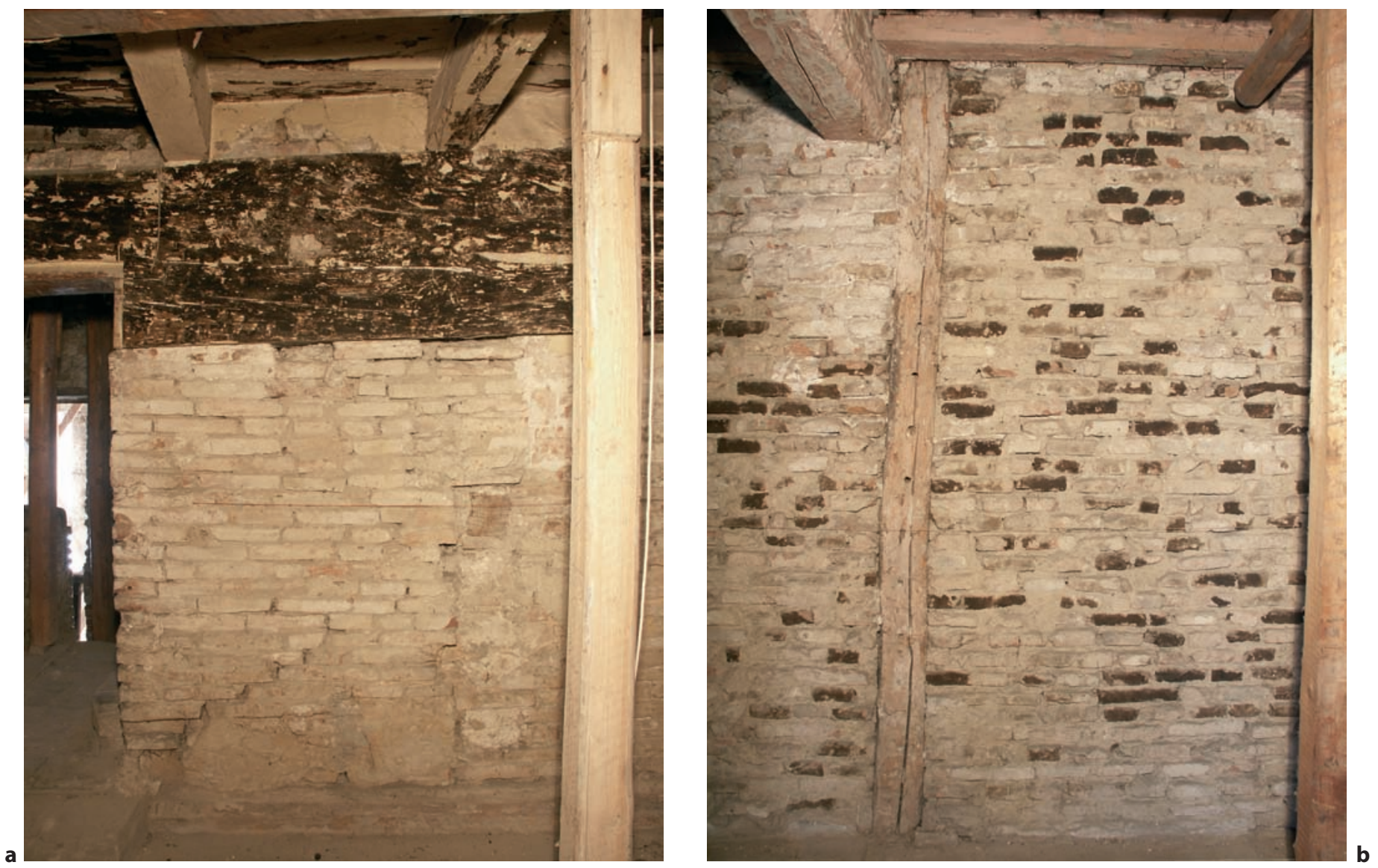

Fig. 11 A-B. Resti degli elementi lignei iniziali e di partizioni interne leggere inglobati nei tamponamenti in muratura successivi

PERIODO C (sec. XIX-XX): intensificazione dell'uso abitativo con modifiche nell'assetto (fase 4)

Si tratta di una fase caratterizzata da murature in foglio e tamponamenti sottili, relativamente recenti, che indicano uno sfruttamento degli spazi del sottotetto a scopo abitativo.

\section{D - edificio nell'attuale conformazione (fase 5)}

L'intervento più recente ha comportato: demolizione $\mathrm{e}$ ricostruzione del tetto, con demolizione di gran parte delle partizioni interne, degli intonaci e degli elementi di arredo fisso. Le opere di consolidamento hanno comportato la formazione di una rifodera in calcestruzzo nella facciata interna, l'esecuzione di iniezioni diffuse ed una rifugatura completa del paramento esterno, oltre all'inserimento nel portico della struttura metallica con pilastri cruciformi ${ }^{10}$.

\footnotetext{
${ }_{10}$ Una limitata attività di documentazione di cantiere relativa a questo intervento, che fa parte della storia recente dell'edificio, ha comportato notevoli difficoltà di ordine sia conoscitivo che operativo.
}

\section{DEGRADO COME PERDITA DI INTEGRITA' E COME AGGIUNTA DI SIGNIFICATO: IL RUOLO DEL DEGRADO NELLA PERCEZIONE DELL'AUTENTICITA' MATERIALE}

La Casa dell'Abate è un edificio in cui il degrado è complesso, oltre che diffuso. Si compongono infatti fenomeni di diversa natura, con forme di degrado proprio della materia, in stato di abbandono da decenni e sottoposta ad un lungo processo di invecchiamento (fig. $13 \mathrm{~A}$ ); effetti conseguenti ad un degrado strutturale conclamato, per cui il dissesto è effetto di un danno strutturale ma anche concausa di un cinematismo di degrado (fig. 13 B). Infine si osservano forme di degrado antropico, correlate all'utilizzo di materiale incompatibile ed a modalità di intervento invasive e non reversibili (utilizzo di malte ad alto contenuto di sali, cemento e resine), principali responsabili dei danni osservabili sulle superfici e nelle strutture dell'edificio (fig. 13 C).

La fenomenologia del degrado ha dunque qui due componenti che agiscono su piani diversi e richiedono un approccio differenziato: quella materiale, da cui dipende la permanenza fisica delle parti e richiede azioni di contrasto; 


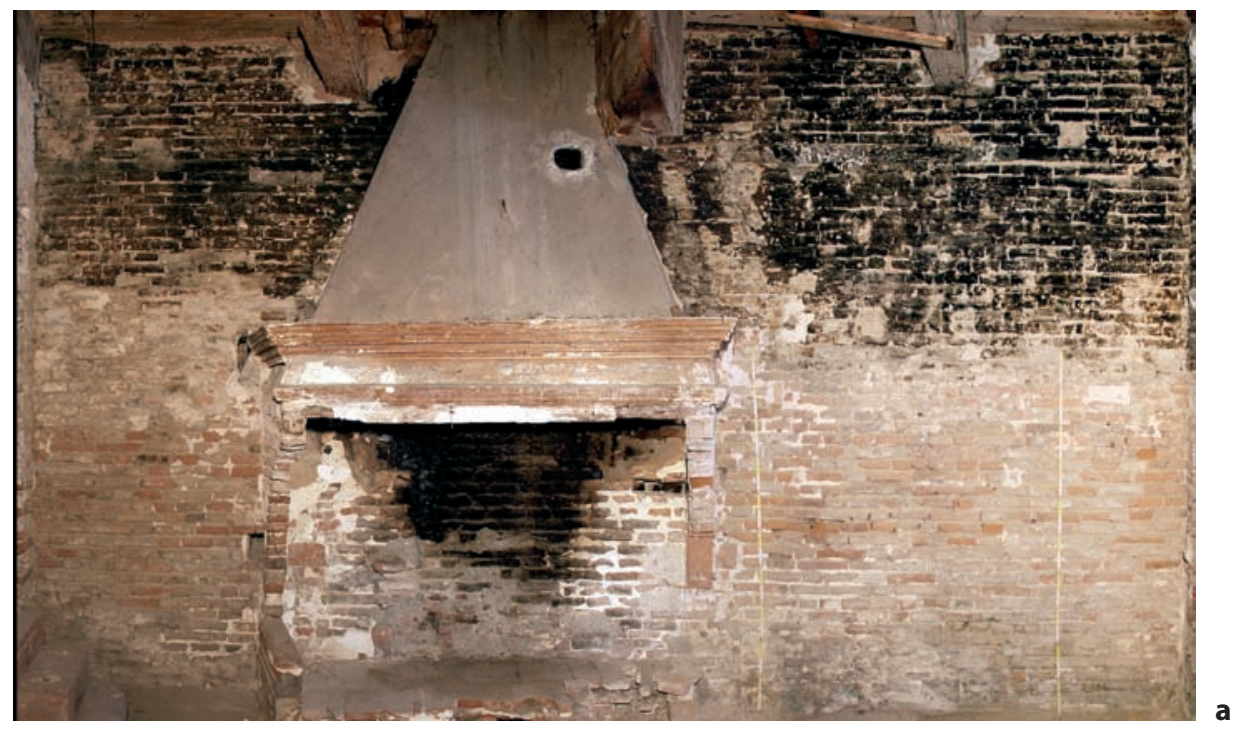

Fig. 12 A-B. Primo piano: un camino ed il corpo scala addossati alla muratura iniziale e all'arco d'ingresso

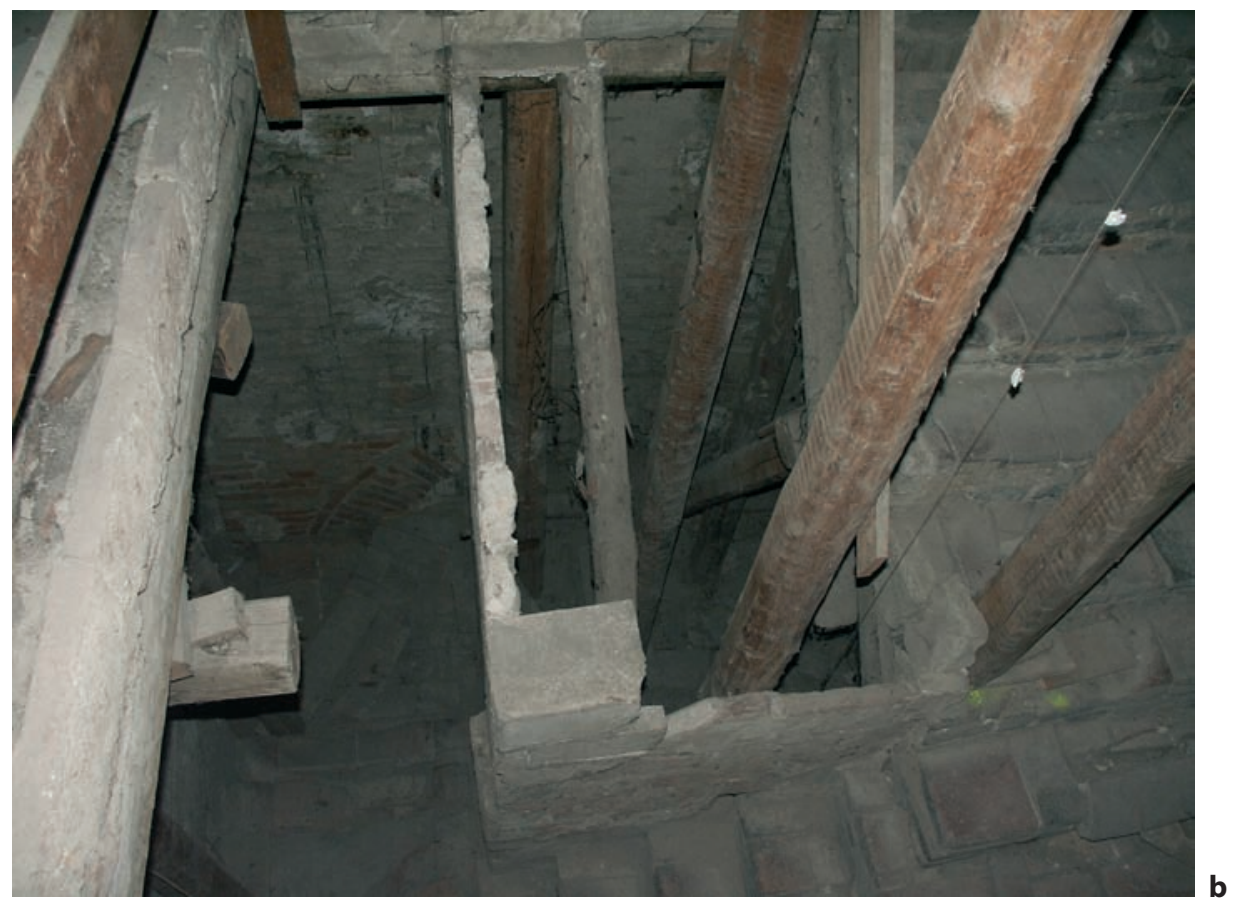

quella formale, che influisce sulla percepibilità delle parti antiche, spesso private della loro carica evocativa da interventi di rinnovo estenuati.

$\mathrm{Da}$ un lato dunque le forme di degrado proprio della materia rappresentano un problema conservativo; d'altra parte per alcuni aspetti esse costituiscono anche un elemento della fisionomia dell'edificio, determinante per la sua estetica. Questo carattere di antichità, legato alla percezione del tempo attraverso i segni materiali, è raggiunge grande intensità proprio nella facciata interna non restaurata e nei pilastri lignei, che il tempo ha eroso portando in rilievo la trama delle venature della superficie sottoposta ad un secolare processo di invecchiamento naturale.

La permanenza della materia autentica - intendendo qui per autenticità l'accezione di "genuinità» della materia non manipolata - è la sola condizione capace di conservare inalterata, a dispetto della mancanza di integrità, la naturalezza delle evidenze stratigrafiche e della leggibilità dei nessi.

In questo edificio l'autenticità richiama infatti, in senso ruskiniano, la naturalezza di contesti materiali intonsi, che conservano la spontaneità di una composizione equilibrata anche nel degrado. La cui consistenza non è 

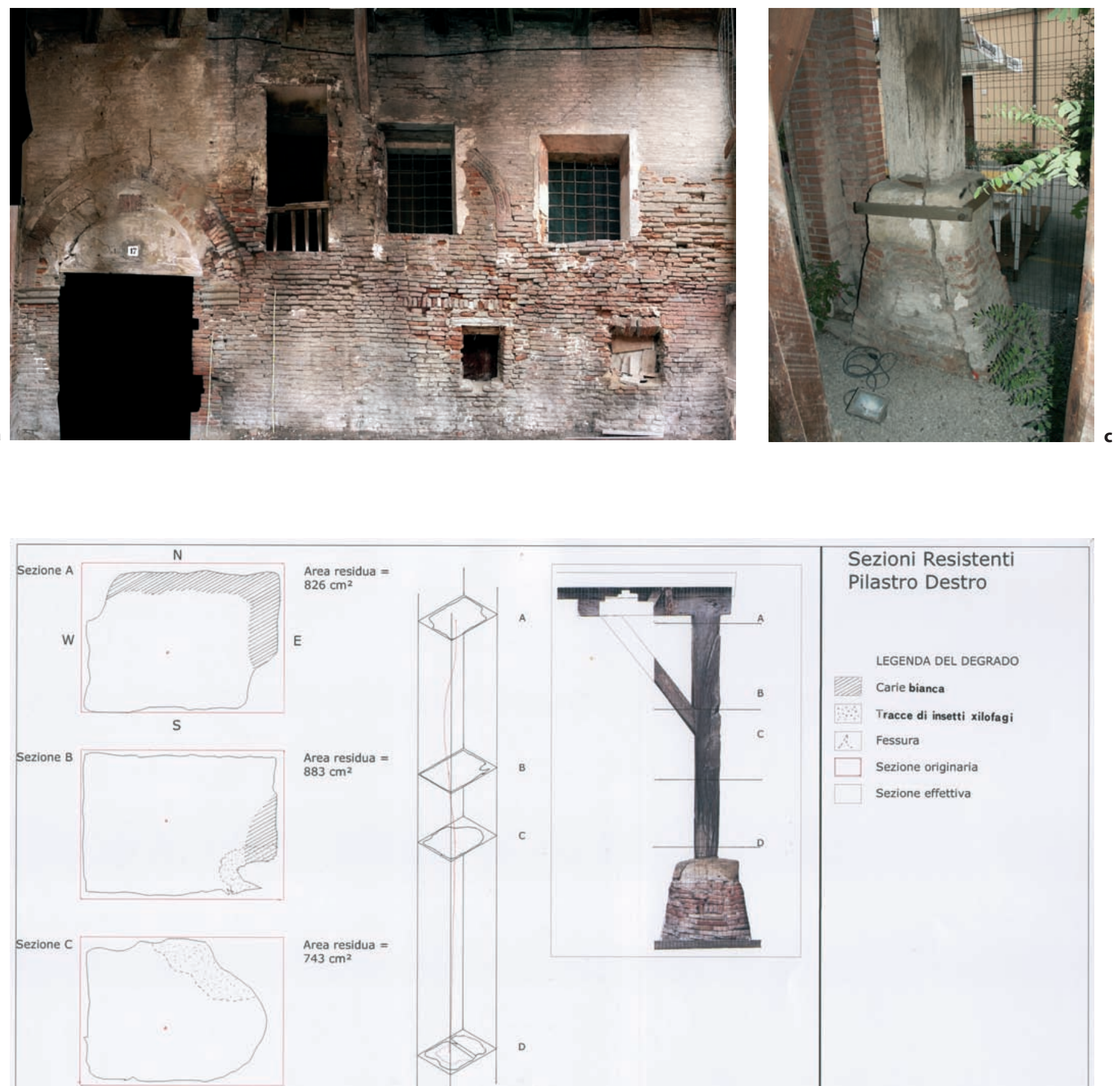

Area residua $=$ $883 \mathrm{~cm}^{2}$
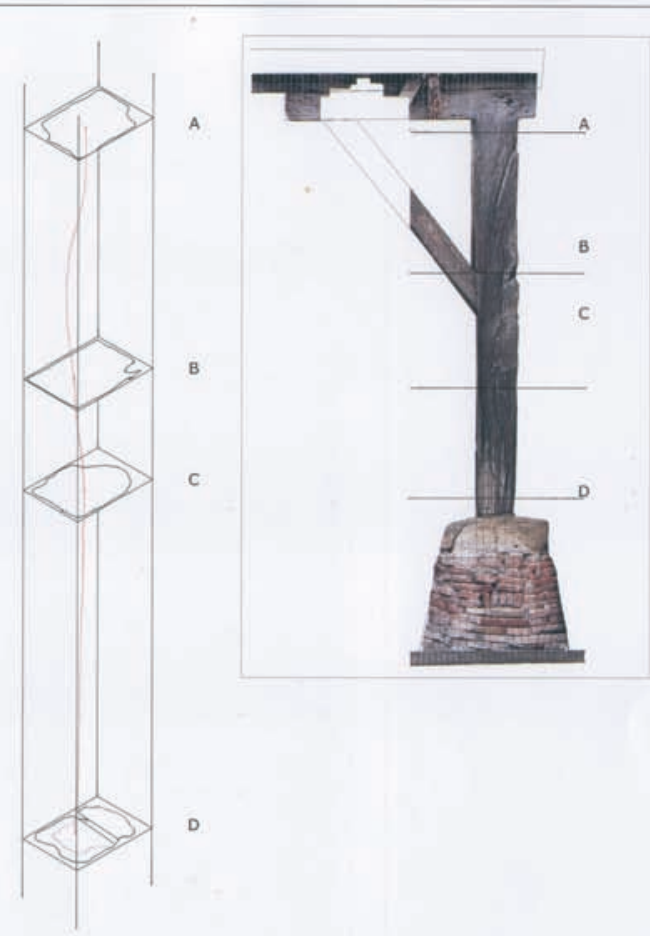

\section{Sezioni Resistenti Pilastro Destro}

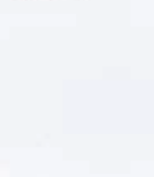

Area residua $=$ $743 \mathrm{~cm}^{2}$

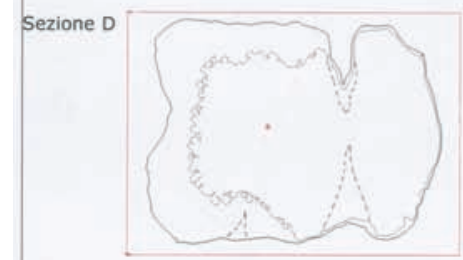

Area originaria $\quad=28 \times 38=1055 \mathrm{~cm}^{2}$ Area residua $=728 \mathrm{~cm}^{2}$ svantaggiosa Area sez.1

$=462 \mathrm{~cm}^{2}$
$=213 \mathrm{~cm}^{2}$

$\sigma=20120 \mathrm{~kg} / 228 \mathrm{~cm}^{2}=27 \mathrm{~kg} / \mathrm{cm}^{2}$ $\sigma=20120 \mathrm{~kg}^{2} 497 \mathrm{~cm}^{2}=40 \mathrm{~kg}^{2} \mathrm{~cm}^{2}$

Area sez.2

$\sigma=10060 \mathrm{Kg} / 213 \mathrm{~cm}^{2}=47 \mathrm{~kg} / \mathrm{cm}^{2}$

Considerando una sezione media $\mathrm{Am}=479 \mathrm{~cm}^{2}$, si ottiene $\sigma=37 \mathrm{~kg} / \mathrm{cm}^{2}$

b

Fig. 13. A. La facciata interna non restaurata trasmette un forte senso di antichità anche grazie al degrado naturale dei materiali. B. Degrado e dissesto interagiscono nelle strutture lignee più antiche (elaborato grafico degli studenti del Laboratorio Integrato di Restauro Architettonico, luav-Venezia, a.a.2002-2003, Prof. F. Doglioni, F. Focacci, F. Laner). C. L'utilizzo di malte cementizie e metallo ossidabile nelle opere di consolidamento degli anni ' 60 ha causato la disgregazione della muratura degli antichi plinti 
Fig. 14. Simulazioni dell'impatto fisico e percettivo di diversi tipi di serramento e della rimozione dei sostegni in muratura dal portico (elaborato grafico dal Laboratorio Integrato di Restauro Architettonico, luavVenezia, a.a.2002-2003, Prof. F.Doglioni, Prof. F.Focacci,

Prof.Laner)

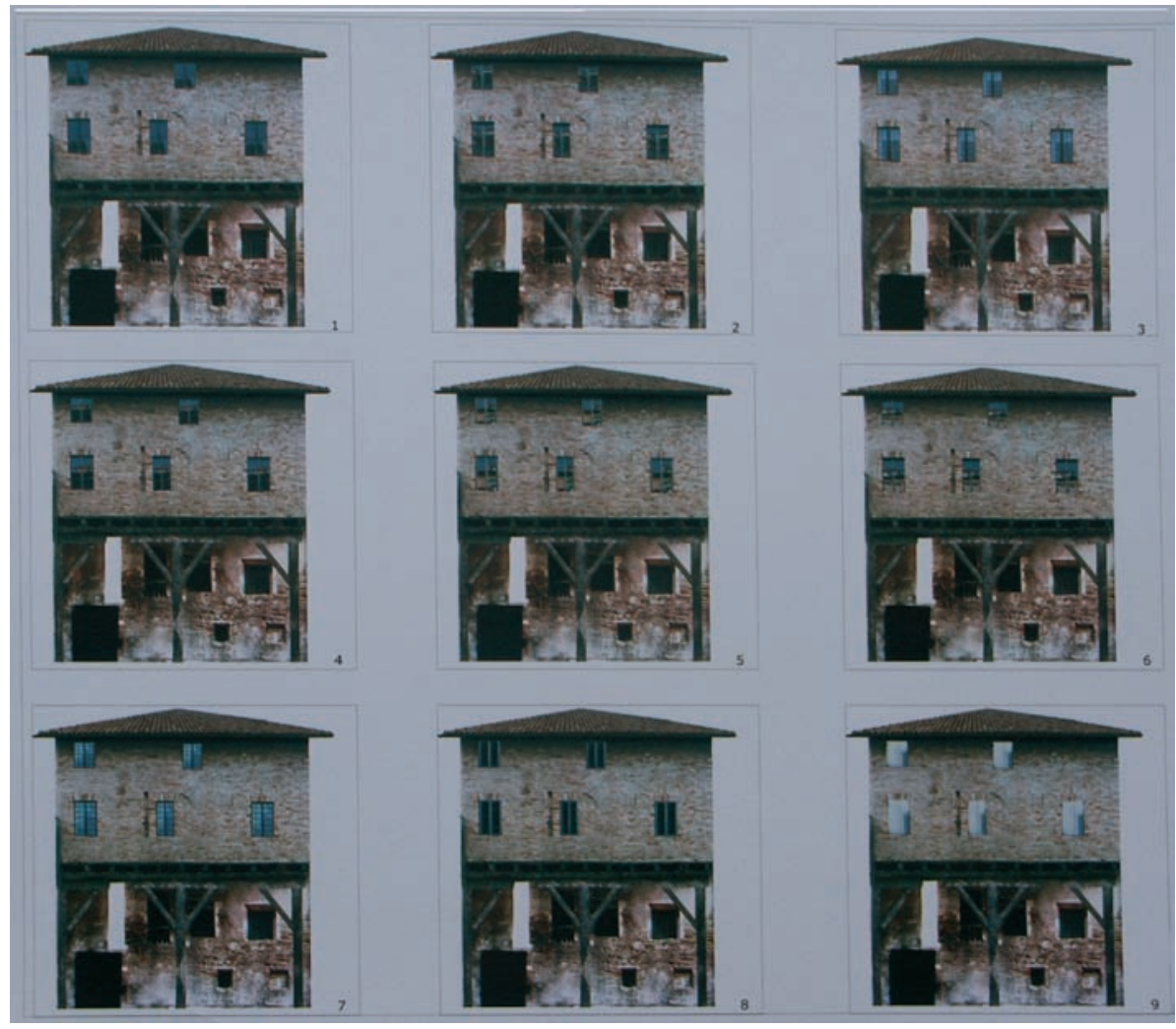

ancora stata congelata da gesti compositivi, o alterata da interventi responsabili della rigidezza o artificialità delle forme "che conservano ben poco della natura virente.» ${ }^{11}$

Si tratta di un edificio attraverso il quale si percepisce una temporalità complessa nella contiguità di parti quasi fossilizzate ed elementi autonomi di nuova formazione: alla continuità temporale ed alle successioni d'uso recepibili nelle stratificazioni organiche conservate, si contrappongono visioni temporali spezzate, frammentate, suggerite dai lacerti ormai disarticolati della struttura lignea iniziale, dalle brusche interruzioni del tessuto murario, dovute a ricuciture recenti e sommarie.

E' qui in un certo senso sperimentabile, attraverso una percezione visiva ma anche tattile, l'irreversibilità in due dei suoi aspetti più significativi: l'irreversibilità dei naturali processi di invecchiamento, percepibile in termini di temporalità, come senso di durata del manufatto nel tempo.

D'altro lato, si verifica l'irreversibilità degli esiti dell'ultimo intervento, concepito secondo una logica di rinnovo e cancellazione dei segni di degrado che ha prodotto un'estrema regolarità ed omologazione delle linee e delle superfici. Una profonda modifica della fisionomia dell'insieme, che trasmette la percezione di contrasto e di

${ }^{11}$ J. Ruskin, «La natura del Gotico», (1852), 1990, pag. 232. negazione dell'idea stessa di antichità, più consona alla natura dell'edificio.

La compresenza di parti così diversamente connotate fa della Casa dell'Abate un edificio in cui il degrado assume un significato concettuale, di cui non è possibile prescindere in ambito operativo. Dove anche una legittima soluzione al degrado, come l'integrazione dei giunti di malta, non può essere intesa come semplice rimedio tecnico ma va adottata come vera e propria scelta progettuale, che richiede una precisa calibrazione rispetto al significato fisico, simbolico, stratigrafico della parti antiche superstiti.

\section{ALCUNE RIFLESSIONI SULL'IMPORTANZA DI UN UTILIZZO COMPATIBILE CON LA CONSERVAZIONE DELLA LEGGIBILITÀ STRATIGRAFICA}

La leggibilità, nei suoi diversi aspetti, assume in questo edificio un ruolo centrale: alla lettura stratigrafica dei diversi passaggi della vita del manufatto, si aggiungono considerazioni sulla negazione della leggibilità, come esito constatato di un intervento e come orizzonte di rischio, per la consapevolezza che ogni futuro intervento, introducendo elementi di rinnovo, può comportare ulteriori riduzioni della materia e con essa delle potenzialità testimoniali dell'intero manufatto.

Ovviare al rischio di una perdita totale dell'autentici- 
tà, per graduali ma continue sostituzioni materiali, diventa una priorità progettuale, cui va subordinata la scelta della destinazione d'uso e che richiede di valutare possibilità di fruizione non intensive, probabilmente minimali, ma compatibili con la permanenza del carattere stratificato e dell'identità complessa che nel tempo l'edificio ha assunto (fig. 14).

La leggibilità diventa così obiettivo e tema progettuale, non intesa come pretesto per operare selezioni arbitrarie, piuttosto come strumento concettuale di un intervento conservativo, che mira a rendere comprensibile la frammentarietà degli elementi riconducibili alla fase più antica - che sono spesso residui disarticolati e privati dei nessi più significativi. Rinunciando a propositi ricostruttivi, la coerenza perduta dell'assetto iniziale va fatta «riemergere» percettivamente, in una lettura guidata dei lacerti.

Lo sviluppo di questi temi-problemi, legati alla singolare condizione delle superfici, ha consentito, al di là degli esiti finali della lettura, di evidenziare l'utilità di una sperimentabilità metodologica, sia in relazione alla specifica finalità conoscitiva che in ordine alle questioni concettuali legate al progetto.

In questo senso, il lavoro condotto rappresenta in parte un esito di tipo operativo, cioè la segnalazione di un percorso in cui modalità di indagine e fini progettuali coincidono, nella volontà di un continuo interrogarsi davanti alla materia antica.

Il tema che si delinea in questo caso è quello del rapporto fra leggibilità stratigrafica e conservazione dell'autenticità. In un edificio antichissimo ma fortemente alterato, la stratigrafia si piega alla necessità di adattarsi a diverse condizioni di lettura e l'analisi stratigrafica perde a tratti la connotazione di strumento risolutivo assoluto. Tuttavia il suo ruolo può risultare più articolato e significativo se da un piano puramente analitico si passi a considerare diversi possibili approcci in relazione al progetto di restauro. La leggibilità stratigrafica è in questo senso un prezioso indicatore, capace di registrare l'entità delle alterazioni indotte dal restauro e di suggerirne una riduzione degli impatti. Diventando anche un criterio tecnico-culturale di orientamento del progetto, che nel caso specifico mira a conservare $\mathrm{i}$ resti come reliquie ma anche a correggere, rendendolo meno stridente, il divario con le parti rinnovate. L'intendere la leggibilità stratigrafica come tensione ideale al controllo delle trasformazioni nell'intervento mette in luce la fondamentale valenza conservativa dello strumento stratigrafico, ove organicamente incluso nella concezione del progetto di restauro.

\section{Bibliografia}

AA.VV., "La casa dell'abate di Nonantola detta il Palazzaccio», in G. Tampone, G. Trota (a cura), "Legno nel Restauro e restauro del legno», Atti del Congresso Nazionale-catalogo sezione espositiva, Firenze, 1983.

A. BELLINI, "La superficie registra il mutamento: perciò deve essere conservata», in 'Le superfici dell'architettura - Le finiture', Atti del convegno di Bressanone, 1990, Arcadia, 1990.

F. BocChI, A. BENATI, «I portici di Bologna e l'edilizia civile medioevale», Grafis, Casalecchio di Reno, 1990.

V. BusaCchI, "Il palazzo dell'abate di Nonantola in San Giovanni in Persiceto», Persiceto (1986), ristampa, Bologna, 1959.

F. Doglioni, «Importanza dell'intonaco per la conservazione delle autenticità edilizie nel restauro architettonico", in 'International workshop on Conservation of architectural surfaces: stone and wall covering', atti a cura di G. Biscontin e L. Graziano, Venezia 1993.

F. Doglioni, «Stratigrafia e Restauro», Lint, Trieste, 1997.

G. ForNI, «Memorie storico-legali sulla Partecipanza di S. Giovanni in Persiceto", Persiceto (1896), ristampa, Bologna, 1959.

L. Gutiérrez, L. Maggioli, A. Nicoli, «Ipotesi sull'originaria destinazione della Casa dell'abate o "Palazzaccio" di San Giovanni in Persiceto", in 'Strada Maestra”n. 3, Forni, Bologna, 1970.

V. HugO, «La bande noire» (1823) in AA.VV., «Dossier: per i duecento anni di Victor Hugo", in 'Antkh', n. 33, marzo 2002, pp. 2-93.

G. RIVANI, «Le case romaniche. Aspetti e singolarità dell'architettura bolognese nel periodo romanico», Strenna storica bolognese XIV, Bologna, 1966.

J. RUSKIN, "La natura del Gotico», in "Le pietre di Venezia», (1852), Bur, Milano, 1990.

Recibido: 16 de julio de 2008

Aceptado: 13 de enero de 2009 